\title{
Der Fremde als Katalysator für Inventionen: kommunale Innovations- förderung auf Grundlage der Unbestimmbarkeit von Handlungsfolgen
}

\section{Peter Dirksmeier, Berlin}

\section{Einleitung}

Die Gesellschaft ist auf Neuerungen angewiesen, um angemessen auf ungewohnte Problemlagen und Situationen reagieren $\mathrm{zu}$ können. Eine klassische Quelle der Entstehung von Neuem ist der Kontakt zwischen Fremden, dessen Regelhaftigkeit zugleich den Beginn der Stadt markiert (WeBer 1999: 115). In der Semantik des Fremden kondensiert die Möglichkeit des Einführens oder Entstehens von Neuerungen für die Gesellschaft in einer Figur. Der Fremde ist nach Simmels klassischer Definition die Symbiose aus der Nähe des habituell wie räumlich Fernen (SimmeL 1992: 764). Simmel nimmt mit dieser Definition mindestens zwei paradigmatische Bestimmungen vor: Der Fremde ist aufgrund der Kombination von Nähe und Entferntheit objektiv und folglich zu Erkenntnisleistungen fähig, die den Autochthonen aufgrund ihrer Verbindlichkeiten verwehrt sind. Die Figur des Fremden betont «die Freisetzung von Sachbindungen und die Offenheit für neue Arrangements» (STicHWEH 1992: 296). Neuerungen sind nicht kausal an die Figur des Fremden gekoppelt, aber aufgrund der skizzierten Eigenschaften gehen «immer oder wenigstens sehr häufig» (Sombart 1987: 883) die Impulse von Fremden aus.

Die gegenwärtige Wirtschaftsgeographie diskutiert diesen klassischen Topos der Genese des Neuen aus dem Kontakt zwischen Fremden lediglich im Zusammenhang mit der Entstehung und Förderung von Innovationsprozessen. Analytische wirtschaftsgeographische Arbeiten verzichten weitestgehend auf Fremdheit und rekurrieren stärker auf Konzepte wie z.B. Vertrauen (z.B. MurPhy 2006), geteilte Institutionen (zuletzt FAROLE et al. 2011) oder Adressierbarkeit in Clusterstrukturen (z.B. GrabHer 2001). Fremdheit als Ressource wird dagegen wenig diskutiert. Lediglich Arbeiten im Kontext von gemeindlichen Institutionen der Innovationsförderung (DiRKSMEIER 2009) oder innovativen kommunalen Projekten (IBERT 2003) nutzen das Konzept. Das Handeln zwischen Fremden wird in diesen innovationszentrierten Arbeiten als ein bedeutender Aspekt dieses komplexen Vorgangs erörtert (IBERT 2005, 2009, 2010; MORGAN 2004; STORPER \& VENABLES 2004). Inventionen und Innovationen können demnach aus der geregelten Interaktion zwischen fremden Akteuren resultieren, seien sie fachfremd oder ortsfremd, und aus der Vermittlung zwischen solitären Projekten oder Institutionen durch Fremde (zu letzteren IBERT 2009).

Das Ziel des Beitrags besteht in der theoretischen Zusammenführung der Konzepte von Fremdheit und Kontingenz, um die in einer solchen Zusammenlegung vermuteten analytischen Potentiale für die Wirtschaftsgeographie und kommunale Innovationsförderung auszuloten. Fremdheit zielt dabei auf die höhere Wahrscheinlichkeit des Abweichens von gewohnten Handlungsmustern, Kontingenz hingegen problematisiert stärker die Regelhaftigkeit des Handelns als den Ausschluss von Handlungsoptionen. Der Beitrag entwickelt daher die These, dass die Interaktion zwischen Fremden an eine unabwendbare dreifache Kontingenz gekoppelt ist. Das Prinzip der Kontingenz verneint eine Kausalität zwischen zwei unterschiedlichen Ereignissen. Bezieht man diesen Sachverhalt auf das menschliche Handeln bedeutet dies, dass identisches Handeln verschiedene Kontinuitäten in verschiedenen zeitlichen und räumlichen Kontexten haben kann (BATHELT \& GlǘKLER 2003: 127). Diese Unvoraussagbarkeit der handlungslogischen Konsequenzen lässt sich nicht über kontextspezifische Konzeptionen der Intentionen und Wirkungen des menschlichen Handelns kontrollieren. Eine Interaktion als Spezialfall des Handelns ist zweitens immer an das Auftreten von doppelter Kontingenz gekoppelt, die aus der gegenseitigen Erwartungsunsicherheit der Akteure in Bezug auf ihre wechselseitigen Handlungen und komplementären Reaktionen resultiert (PARSONs et al. 2001: 16). Die dritte Kontingenzebene entsteht schliesslich aus der Unbestimmtheit des Fremden, die sich aus dem Sein ergibt. Daher bedeutet der Fremde Möglichkeiten, aber u.U. ebenfalls Risiken für die Gesellschaft, die ohne ihn gehemmt wären (SтіснWEH 1997: 165).

Der Aufsatz analysiert zunächst die Kontextungebundenheit des Handelns und die ontische Unbestimmtheit des Fremden als wesentliche Merkmale der Interaktion von Fremden. Die resultierende dreifache Kontingenz wird als eine Triebfeder in der Erzeugung von Neuem interpretiert (Kapitel 2). Der Beitrag ordnet dieses Argument anschliessend in den Forschungskontext ein und skizziert darauf aufbauend Möglichkeiten für die kommunale Innovationsförderung, die Fremde gezielt zusammenbringt. Die Qualitäten der beteiligten Akteure generieren in diesem Fall die drei Kontingenzebenen des Fremdhandelns 
und stellen damit Möglichkeiten bereit, aus denen Neuerungen als Zufälle resultieren (Kapitel 3). Der Beitrag schliesst mit einer Diskussion des Arguments für die kommunale Innovationsförderung (Kapitel 4).

\section{Was passiert, wenn Fremde handeln?}

Die Entstehung von Neuerungen ist eng an Kontingenz als Möglichkeitsraum des Handelns in der Gesellschaft gekoppelt. Handlungen sind in einem allgemeinen Sinne einem System zurechenbare Selektionsleistungen (LuHmann 1987: 160). Die Konstitutionsleistungen der Subjekte, ob Fremde oder nicht, die Welt so und immer gleichzeitig anders zu deuten und entsprechend ihrer Deutungen situativ zu handeln, stellen damit eine wichtige Quelle von Kontingenz dar (WeICHHaRt 2003: 23). Kontingenz bezeichnet «jene spezifische Unbestimmtheit, in der etwas weder notwendig noch unmöglich ist und sich darin als wirkliche Alternative manifestiert» (MAKropoulos 2004: 371). Kontingenz ist somit dem Sozialen nicht vorausgesetzt, sondern wird erst in der Gesellschaft definiert. Mit dieser Feststellung sind Vorgänge oder Ereignisse kontingent, die von etwas Gegebenen im Rahmen einer möglichen Andersheit divergieren (LÜBBE 1998: 35). Kontingenz ist keine natürliche, unabhängig von dem sozialisierten Menschen gegebene Tatsache oder anthropologische Konstante, sondern ein historisch variables Produkt der Selbstreflexion einer Gesellschaft. Sie ist daher keinesfalls gleichzusetzen mit dem Begriff des Zufalls. Kontingenz ist der sich logischontologisch erschliessende Raum von Realisierungschancen unterscheidbarer Ereignisse, die aber nicht notwendigerweise eintreten müssen (BUBNER 1998: 7). Zufall ist hingegen die Verwirklichung eines Ereignisses als ex-post-Bestimmung in diesem Kontingenzrahmen möglicher Ereignisse. Die Bedingungen, die sich für das Auftreten des Zufalls stellen, sind zum einen die Grundlosigkeit seines Eintretens und zum anderen das Eintreten selbst. Der «Zufall ist grundlos fixierte Kontingenz» (Bubner 1998: 7) und wird nach dem Ereignis erst als zufällig erkannt. Der Kontingenzraum bildet dagegen die Grenze menschlicher Handlungsoptionen. Jede einzelne Handlung ist eine Realisierung einer einzigen Möglichkeit auf Kosten aller anderen denkbaren Möglichkeiten des Kontingenzrahmens (HAHN 1998: 495).

Ein grundlegendes Problem der Handlungstheorie liegt daher in der Unterscheidung zwischen Ereignis und Handeln oder anders ausgedrückt, zwischen dem, was ein Akteur macht und was ihm passiert (FranKFURT 1978: 157). Mit dieser Unterscheidung ist das Verhältnis von Absicht zu Handlung berührt. Nicht alle Handlungen sind zugleich absichtliche Handlungen. Vielmehr können unbeabsichtigte Handlungen, die ungewollt oder misslungen sind, wiederum unintendierte Folgen haben und dabei Bestehendes modifizieren. Die unabsichtlichen Handlungen sind existent, aber ihr Bestehen verdankt sich einer vorgängigen intendierten Handlung (LeIST 2007: 524). Handlungen weisen eine Unvoraussagbarkeit ihrer handlungslogischen Kontinuitäten auf, die ihre Kontingenz begründet. Der Handlungskontext strukturiert entlang der Intentionen des Handelns den Möglichkeitsraum sich realisierender intendierter und nicht-intendierter Handlungsfolgen. Kontingenz ermöglicht somit das Handeln als ein Festlegen von Wirklichkeit, die noch nicht ist, ob absichtlich oder unabsichtlich (BUBNER 1998: 7). Als soziale Praxis erscheint mithin die Bedeutung, die ex post aus dieser ersten Kontingenzebene individuellen und kollektiven, absichtsvollen und unintendierten Handelns entsteht (Alexander 2004: 527).

Die zweite logische Bedingung der Möglichkeit des sozialen Handelns bildet Kontingenz in Form doppelter Kontingenz in der Interaktion. Interaktion bildet einen Spezialfall sozialen Handelns als ein Mitteilungshandeln unter der Bedingung wechselseitiger Wahrnehmbarkeit der Handelnden (KIESERLING 1999: 153). Interaktion und Handeln fallen unter dieser Prämisse empirisch zusammen als «Konkretisierung von Kommunikation» (HeIdenescher 1992: 442) in einer Situation, wobei, wie LuHMANN (1987: 193) betont, beide Begriffe nach wie vor theoretisch $\mathrm{zu}$ unterscheiden sind. Jeder Interaktion als Mitteilungshandeln ist demnach eine doppelte Kontingenz inhärent, die als gegenseitige Erwartungsunsicherheit in Bezug auf die Handlungen des anderen entsteht. Die Unprognostizierbarkeit der handlungslogischen Konsequenzen und Intentionen von Ego erzeugen einen Kontingenzraum für Alter, der auf der Handlung von Ego beruht und wiederum von diesem begrenzt wird, in seinen Grenzen aber signifikant von dem Kontingenzraum Egos abweicht (PARsons et al. 2001: 16). Alters Handeln erweist sich folglich als eine Selektion aus mannigfachen Möglichkeiten, die in dem Kontext vollzogen wird, dass Egos Handeln genauso eine solche Selektion bedeutet. Doppelte Kontingenz weist demnach einen Handlungsaspekt als Setzen von einer Wirklichkeit in einem jeweils gegebenen Kontingenzraum auf und bezeichnet gleichzeitig die Tatsache, dass Ego und Alter die Welt auf unterschiedliche und damit kontingente Weise erleben können (HaHN 1998: 500).

Die Interaktion zwischen Fremden fügt den skizzierten zwei Kontingenzebenen eine dritte hinzu. Der Fremde selbst ist qua definitionem kontingent. Er ist von seiner ontischen Qualität her unbestimmt (STICHWEH 1997: 165). Der Fremde generiert so einen erweiterten Kontingenzraum, in dem Handeln sich vollziehen kann, da er selbst als soziale Figur neue Möglichkeiten für 


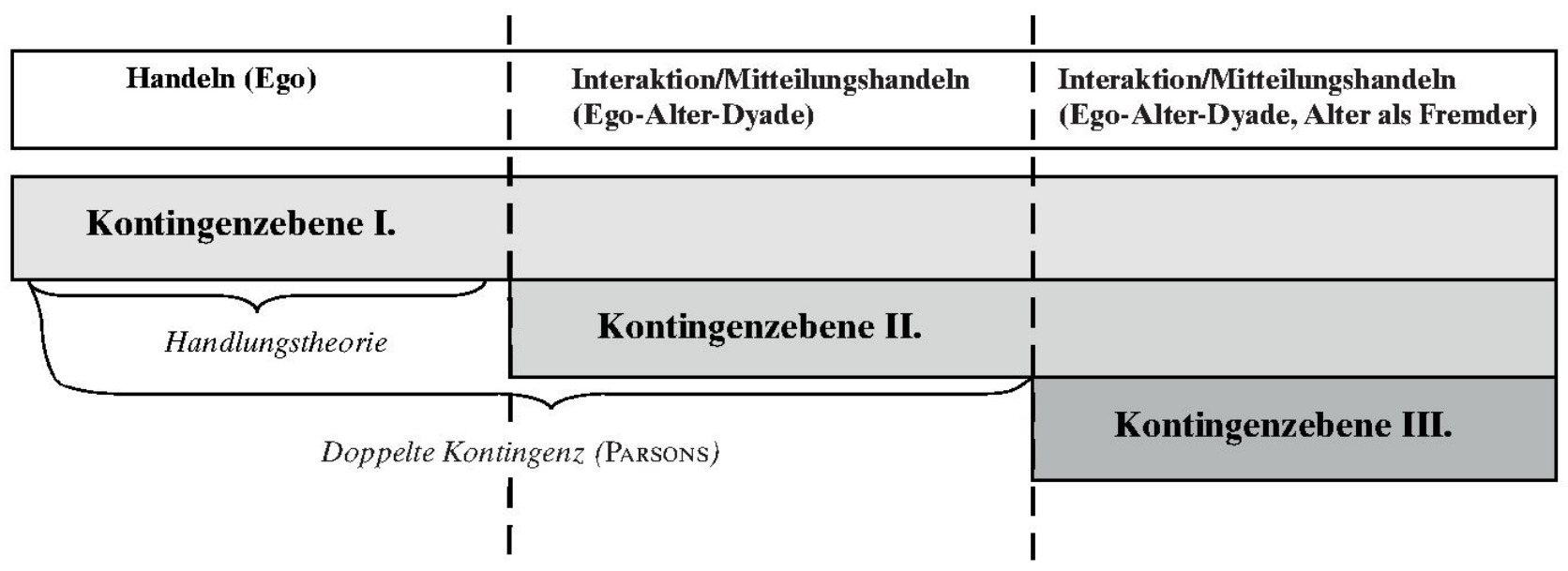

Abb. 1: Die drei Kontingenzebenen des Fremdhandelns The three contingency levels of foreign interaction Les trois dimensions de contingence de l'action entre étrangers Quelle: eigene Darstellung

die Gesellschaft bedeutet, die ohne die Existenz des Fremden unerreichbar und damit aus dem gegebenen Kontingenzraum des Handelns der Gesellschaft ausgeschlossen wären (StichweH 1997: 165). Jede soziale Beziehung mit dem Fremden, die sich in dem Kontingenzraum der gegebenen Handlungsmöglichkeiten manifestiert und konkretisiert, etabliert ihrerseits wiederum «eine Mehrzahl von Unbestimmtheiten als neue Möglichkeiten, die es vorher nicht geben konnte» (HONDRICH 1985: 75). HoNDRICH spricht daher von einem «Wachstumsvorsprung der Unbestimmtheit gegenüber der Bestimmtheit» (1985: 75, Hervorhebung im Original). Die Fremdheit des Fremden koppelt demnach eine zusätzliche Kontingenzquelle an die unabhängig vom Fremden bestehende doppelte Kontingenz der Interaktion als Mitteilungshandeln. Die Interaktion zwischen Fremden unterscheidet sich dagegen von der Interaktion mit Bekannten, indem die Möglichkeit der Erwiderung eingeschränkt ist, d.h. eine temporäre Unausgewogenheit der Austauschbeziehung wahrscheinlich nicht ausgeglichen wird. Zeitweilige Unausgeglichenheiten in den Reziprozitätsbeziehungen zwischen Bekannten fallen dagegen weniger ins Gewicht, solange der Austausch aufrechterhalten bleibt (SHAPIRo 1980). Der Fremdkontakt lässt somit eine Unsicherheit entstehen, da die beteiligten Akteure nicht davon ausgehen können, dass eingegangene Schuldverhältnisse ausgeglichen werden. Sie sind strukturell gehalten, den mitunter einmaligen Kontakt mit einem Fremden für sich ökonomisch zu nutzen. Das Risiko des Fremdkontakts ist folglich eine Optionssteigerung des eigenen Handelns unter der Bedingung dreifacher Kontingenz. Abbildung 1 stellt die drei unterschiedlichen Kontingenzebenen schematisch dar.
Die Gesellschaft hat im Wesentlichen zwei Möglichkeiten des Umgangs mit den drei skizzierten Kontingenzebenen des Fremdkontakts. Sie kann versuchen, diese weitestgehend einzudämmen, indem sie den Kontakt zwischen Fremden als gefährlich betrachtet und zu verhindern sucht. Sie kann den Fremdkontakt genauso als eine riskante Form des Handelns begreifen und versuchen, seine Kontingenz zu institutionalisieren und in Wert zu setzen. Der Umgang mit dem Fremden ist eine Chance, Gewinne z.B. über den Warentausch oder das Zulassen von Neuerungen $\mathrm{zu}$ erzielen, die bewusst gewählt oder ausgelassen werden kann (StichweH 1992: 303). Auf spieltheoretischen Modellen basierende Studien zeigen in diesem Zusammenhang einen stochastischen cooperator's advantage für Akteure, die solcherart riskante Austauschprozesse mit Fremden eingehen. Dies gilt sowohl für kurzfristige ökonomische Vorteile (MACY \& SKvoreTz 1998) als auch in langfristiger evolutionärer Perspektive (Bowles 2009). Diese langfristig sich einstellenden Gewinne könnten der Grund für das, mit Ausnahme von nur wenigen Gruppen (für eine solche Ausnahme siehe GreIFER 1945), allgemein feststellbare soziale Einlassen auf den Fremden sein.

\section{Handeln zwischen Fremden in praxi: Möglichkeiten für die kommunale Innovationsförderung}

Für die kommunale Innovationsförderung stellt sich das Problem des Verstehens der Komplexität des Innovationsprozesses, der weder von Ökonomie noch Wirtschaftsgeographie vollständig durchdrungen ist. 
«Many traditional concepts in economics and geography fail to properly understand the processes of generating new products and processes» (BATHELT \& GLÜCKLER 2003: 135).

Der Beitrag meint mit Innovation, in Anlehnung an BARTELs (1970: 285) und Dosi (1988), eine prozessuale Abfolge von Invention, verstanden als folgenreiches Zusammentreffen von Informationen und Wissen in einem Akteur oder in einer Organisation sowie deren Diffusion in die Umwelt der Organisation oder des Akteurs, die wiederum an ökonomische Vorteile für den jeweiligen Inventor gekoppelt ist (DiRKSMEIER 2009: 310). Eine solche Innovation ist keine lineare Abfolge, sondern in ihrem Verlauf durch vielfältige Rückkopplungen als Lernprozesse und Anpassungen der beteiligten Akteure und Organisationen gekennzeichnet, die sich nach Markteinführung der Innovation fortsetzen (RosenberG 1982: 122). Aus diesem Grund ist die Kommunikation zwischen Akteuren Voraussetzung für das Zustandekommen von Innovationen (BATHELT \& DEPNER 2003: 130).

Der Aufsatz sieht in der skizzierten dreifachen Kontingenzebene des Handelns von Fremden eine theoretische Ansatzmöglichkeit für die kommunale Förderung von Innovationen und damit gleichzeitig einen Weg des Verstehens dieser von BATHELt \& GLÜCKLER (2003: 135) adressierten Innovationsprozesse. Die kontrollierte Interaktion von Fremden als Katalysator von Innovationsprozessen ist von IBERT in die deutschsprachige Fachdiskussion eingeführt worden (IBERT 2003, 2005). IBERTs These ist, dass Fremde innovative Lösungen auf Grundlage ihrer objektiven Distanz zum Gegenstand entwickeln (IBERT 2010: 190). Diese innovative Kraft entfaltet der Fremdkontakt beispielsweise über die Zusammenführung von Fremden in zeitlich befristeten Projekten (IBERT 2003: 4-5), die fremde Akteure in administrativen Strukturen institutionell kontrolliert interagieren lassen (IBERT 2005: 604). Wirtschaftsförderung greift nicht auf jeden Fremden $\mathrm{zu}$, sondern stellt auf habituell «nahe Fremde» im Sinne SimmeLs (1992: 764-771) ab, die gewünschte Anschlussfähigkeiten an die institutionsinterne Kommunikation aller Wahrscheinlichkeit nach bieten. Mit dieser Adressierung von bestimmten Akteuren als Fremde in der Kommunikation erscheint Fremdheit sowohl als Ressource als auch als ein machtbeladener sozialer Prozess. Der Fremde wird erst mit dieser Anrufung als Fremder im Sinne Butlers (2006: 49) performativ zum Fremden. Damit erscheint die kontingenzerzeugende ontische Qualität der Figur des Fremden ebenfalls als eine aktivierbare ökonomische Ressource, die über die Adressierung eines Subjekts als Fremder von der Wirtschaftsförderung in Wert gesetzt werden kann.

«Das heisst natürlich nicht, dass es den Fremden gar nicht gibt, die Gesellschaft ihn gewissermassen nur herbeidenkt.
Aber es heisst, dass die Gesellschaft den Fremden so selegiert oder formt, wie sie ihn braucht, um Innovationen über Externalisierungen einzuführen» (STіснWEH 1997: 166).

Die selegierbare Fremdheit des Fremden erscheint somit als eine bewusst einsetzbare Quelle für Kontingenz aus der Perspektive der Wirtschaftsförderung.

Die zwei weiteren Kontingenzebenen des Handelns adressiert die in der Wirtschaftssoziologie und -geographie diskutierte Idee der embeddedness (für erstere GranovetTer 1985; für letztere GlückLER 2001). Dieses Konstrukt greift aber inhaltlich zu kurz, da sich die Theoretisierung der Kontingenz des Handelns im embeddedness-Konzept in der Annahme erschöpft, dass Handlungen unintendierte und unvorhersehbare Ergebnisse zeigen können. Die erste handlungstheoretische Kontingenzebene bearbeitet der embeddedness-Ansatz, indem soziale Strukturen als Regeln, Kulturen, Konventionen oder Institutionen den Kontingenzraum des Handelns dahingehend beschränken, dass Egos Handeln für Alter auf Grundlage der Kenntnis dieser Strukturen berechenbar wird (GLÜCKLER 2001: 214). Dies ist exakt die Lösung, die PARsons et al. bereits in den 1950 er Jahren für das Problem der doppelten Kontingenz vorschlagen (PARsons et al. 2001: 16). Der embeddedness-Gedanke verschiebt folglich PARsons' Bearbeitung des logischen Problems der doppelten Kontingenz in der Interaktion auf die Grundbedingung des Handelns selbst - namentlich, dass Handeln, und damit ebenfalls das Handeln von Fremden, Kontingenz als Variabilität der Möglichkeiten voraussetzt. Damit liesse sich letztlich die Entstehung von Neuerungen oder Innovationen nur ex post erklären, als bewusster oder unbewusster Verstoss gegen die strukturellen handlungsleitenden Regeln, der erst nach dessen Vollzug erkennbar würde.

Die kommunale Innovationsförderung könnte zur Inwertsetzung der drei distinkten Kontingenzebenen in der Interaktion von Fremden als eine aussen stehende Vermittlerin zwischen den Netzwerken der an Innovationen in der Regel beteiligten und wechselseitig als Fremde adressierbaren Akteure auftreten, wie z.B. der Kunst- und Kulturszene oder der klassischen Wirtschaft. Die Bedeutung dieser Position des Dritten in einer Dyade ruht in ihrer Eigenschaft, sogenannte weak ties $\mathrm{zu}$ besitzen, d.h. schwache, nur temporär aktivierbare Kontaktmöglichkeiten, die aber eine Brückenfunktion zwischen kommunikativ auf sich selbst verweisende Netzwerke einnehmen können. Informationen diffundieren besonders entlang solcher schwachen Bindungen zwischen verschiedenen Akteursgruppen und fungieren damit als ein Modus der Dissonanzreduktion im Kontakt zwischen Netzwerken (zuerst: GranovetTER 1973: 1367-1368; auch 
BARGATZKy 1981). Die Bedeutung des Dritten in dieser klassischen Position des Vermittlers liegt zunächst darin, die Kontaktchancen für die sich unbekannten Beteiligten $\mathrm{zu}$ generieren und anschliessend den Kontakt in eine sachliche Form zu überführen und damit rational gestaltbar zu machen (LINDEMANN 2006: 97). Solche Interaktionen vollziehen sich als face-to-faceKontakte, z.B. in Seminaren, Messen und Workshops, die als regelmässig stattfindende Veranstaltungen konzipiert sein könnten. Mit einer derartigen Förderstrategie vermittelt die kommunale Innovationsförderung nicht nur Informationsaustausch zwischen den Netzwerken, sondern über die physische Kopräsenz der Akteure wird gleichzeitig das Verstehen dieser Informationen gefördert. Verstehen ist ein Grundproblem in der Vermittlung zwischen Netzwerken. Im Gegensatz $\mathrm{zu}$ einer Information als «Unterschied, der einen Unterschied ausmacht» (BATESON 1985: 582; Hervorhebung im Original) diffundiert das Verstehen nur eingeschränkt über Netzwerkbrücken und bleibt stärker auf face-to-face-Interaktion angewiesen (MoRGAN 2004: 3).

Als entscheidender Punkt der kommunalen Innovationsförderung wird die Verbindung des am Ort vorhandenen technischen Wissens mit besonderen Kompetenzen von Fremden, wie z.B. Künstlern oder anderen Kreativen angesehen. Um die drei Kontingenzebenen des Fremdhandelns $\mathrm{zu}$ involvieren, könnte die Förderinstitution darauf zielen, dass die Akteure zusammen handeln, indem z.B. Workshops und Seminare mit hoher Eigenbeteiligung der Teilnehmer als face-to-face-Veranstaltungen konzipiert werden. Damit wären einerseits die Kontingenzebenen des Handelns berührt, die in Workshops und Seminaren $\mathrm{zu}$ unintendierten Folgeketten führen können. Im Idealfall können diese aus Sicht der Förderinstitution wiederum zu Projekten werden, genauso wie die doppelte Kontingenz der Interaktion (PARsons et al. 2001: 16), deren Aushandlung wiederum Unbestimmtheit erzeugt. Andererseits wäre die Kontingenz des Fremden als unbekannte Kompetenz berührt. Letztlich ist ein bestimmtes Niveau an sozialer Inkommensurabilität der zusammengeführten sich wechselseitig fremden Akteure entscheidend, die dennoch moderiert miteinander zu interagieren beginnen. Welche Möglichkeiten aus der dreifachen Kontingenzebene des Handelns schliesslich realisiert werden und in Inventionen und Innovationen münden, bleibt dem Zufall überlassen.

\section{Fazit}

Mit Blick auf das in diesem Beitrag ausformulierte Argument der drei Kontingenzebenen des Handelns zwischen Fremden kann es für die kommunale Innovationsförderung oder Planungspraxis nicht alleine darum gehen, zufällige Kontakte zwischen sich völlig
Unbekannten herzustellen. Vielmehr liesse sich die Kommunikation zwischen sich fremden Akteuren unterstützen, die trotz ihrer wechselseitigen Wahrnehmung als Fremde genügend Gemeinsamkeiten haben, um von ihrer gegenseitigen Differenz profitieren zu können. In diesem Kontext spannt jede der theoretisch hergeleiteten drei Kontingenzebenen des Handelns einen Möglichkeitsraum für sich ereignende Zufälle auf, die wiederum über Anschlussförderungen in dauerhafte Strukturen überführt werden können. Innovative Innovationsförderung würde diesen Zufällen, die letztlich zu den gewünschten Innovationsprozessen führen könnten, Raum geben, z.B. über Moderationsprozesse.

Der kritische Punkt dieser Argumentation besteht darin, dass die kommunale Innovationsförderung in diesem Fall nicht mehr als ein handelnder Akteur einer dyadisch strukturierten Sozialität auftritt, sondern als die Figur des Dritten, z.B. in der Rolle des Vermittlers oder Schiedsrichters (SIMMEL 1992: 125134) einer solchen von dreifacher Kontingenz geprägten Sozialbeziehung. Die skizzierten drei Kontingenzebenen werden, ähnlich wie das sozialtheoretisch basale Konzept der doppelten Kontingenz, empirisch nicht beobachtbar sein. Dessen ungeachtet können hingegen empirisch beobachtbare Phänomene als die Lösung des zugrundeliegenden Problems der dreifachen Kontingenz der Interaktion zwischen Fremden aufgefasst werden (LindEMANN 2010: 497). Es bleibt zu vermuten, dass solche Phänomene in Innovationsprozesse münden können.

\section{Literatur}

Alexander, J.C. (2004): Cultural pragmatics: social performance between ritual and strategy. - In: Sociological Theory 22, 4: 527-573.

BARGATZKy, T. (1981): Das «Marginal Man» Konzept: ein Überblick. - In: Sociologus. Zeitschrift für empirische Ethnosoziologie und Ethnopsychologie 31, 2: 141-166.

BARTELs, D. (1970): Geographische Aspekte sozialwissenschaftlicher Innovationsforschung. - In: MEcKELEIN, W. \& C. BorcherdT (Hrsg.): Tagungsbericht und wissenschaftliche Abhandlungen. - = Verhandlungen des Deutschen Geographentages 37, Wiesbaden: 283296.

Bateson, G. (1985): Ökologie des Geistes. Anthropologische, psychologische, biologische und epistemologische Perspektiven. - Frankfurt am Main: Suhrkamp. Bathelt, H. \& H. Depner (2003): Innovation, Institution und Region: zur Diskussion über nationale und regionale Innovationssysteme. - In: Erdkunde 57, 2: 126-143.

BATHELt, H. \& J. GLÜCKLER (2003): Toward a relational economic geography. - In: Journal of Economic Geography 3, 2: 117-144. 
Bowles, S. (2009): Did warfare among ancestral hunter-gatherers affect the evolution of human social behaviors? - In: Science 324: 1293-1298.

Bubner, R. (1998): Die aristotelische Lehre vom Zufall. Bemerkungen in der Perspektive einer Annäherung der Philosophie an die Rhetorik. - In: GraEvenitz, G. v. \& O. MARquard (Hrsg.): Kontingenz. - = Poetik und Hermeneutik 17, München: Wilhelm Fink Verlag: 3-21.

Butler, J. (2006): Hass spricht. Zur Politik des Performativen. - Frankfurt am Main: Suhrkamp.

DiRKSMEIER, P. (2009): Innovationsförderung und Verwaltung: das Beispiel der Freien Hansestadt Bremen.In: Raumforschung und Raumordnung 67, 4: 308-317. Dosi, G. (1988): The nature of the innovative process. - In: Dosi, G., Freeman, C., Nelson, R., Silverberg, G. \& L. Soete (Hrsg.): Technical change and economic theory. - London: Pinter:221-238.

Farole, T., Rodríguez-Pose, A. \& M. Storper (2011): Human geography and the institutions that underlie economic growth. - In: Progress in Human Geography 35, 1: 58-80.

FRANKFuRT, H.G. (1978): The problem of action. - In: American Philosophical Quarterly 15, 2: 157-162.

GLÜCKLER, J. (2001): Zur Bedeutung von Embeddedness in der Wirtschaftsgeographie. - In: Geographische Zeitschrift 89, 4:211-226.

GrabHer, G. (2001): Ecologies of creativity: the village, the group, and the heterarchic organisation of the British advertising industry. - In: Environment and Planning A 33, 2: 351-374.

Granovetter, M. (1973): The strength of weak ties. In: American Journal of Sociology 78, 6:1360-1380.

Granovetter, M. (1985): Economic action and social structure: the problem of embeddedness. - In: American Journal of Sociology 91, 3: 481-510.

Greifer, J.L. (1945): Attitudes to the stranger: a study of the attitudes of primitive society and early Hebrew culture. - In: American Sociological Review 10, 6: 739745.

HaHn, A. (1998): Kontingenz und Kommunikation. In: Graevenitz, G. v. \& O. Marquard (Hrsg.): Kontingenz. - = Poetik und Hermeneutik 17, München: Wilhelm Fink Verlag: 493-521.

Heidenescher, M. (1992): Zurechnung als soziologische Kategorie. Zu Luhmanns Verständnis von Handlung als Systemleistung. - In: Zeitschrift für Soziologie 21, 6: 440-455.

Hondrich, K.O. (1985): Begrenzte Unbestimmtheit als soziales Organisationsprinzip. - In: Neue Hefte für Philosophie 24/25: 59-78.

Ibert, O. (2003): Projekte und Innovation. Projektorientierung in der Entwicklungsplanung als Antwort auf das Problem der Organisation von Innovation. - In: Raumforschung und Raumordnung 61, 1-2: 3-12.

IBERT, O. (2005): Wie lassen sich Innovationen planen? In: Informationen zur Raumentwicklung 9/10:599-607.
IBERT, O. (2009): Innovationsorientierte Planung und das Problem des episodischen Lernens. - In: DannEnBERG, P., KöHLER, H., LANG, T., UtZ, J., ZaKirova, B. \& T. ZimMERMANN (Hrsg.): Innovationen im Raum - Raum für Innovationen. $-=$ Arbeitsmaterial der Akademie für Raumforschung und Landesplanung 348, Hannover: 18-28.

IBERT, O. (2010): Relational distance: sociocultural and time - spatial tensions in innovation practices. - In: Environment and Planning A 42, 1: 187-204.

Kieserling, A. (1999): Kommunikation unter Anwesenden. Studien über Interaktionssysteme. - Frankfurt am Main: Suhrkamp.

LeIst, A. (2007): Über Kontexte zu Handlungen. - In: Deutsche Zeitschrift für Philosophie 55, 4: 521-544.

Lindemann, G. (2006): Die Emergenzfunktion und die konstitutive Funktion des Dritten. Perspektiven einer kritisch-systematischen Theorieentwicklung. - In: Zeitschrift für Soziologie 35, 2: 82-101.

Lindemann, G. (2010): Die Emergenzfunktion des Dritten - ihre Bedeutung für die Analyse der Ordnung einer funktional differenzierten Gesellschaft. In: Zeitschrift für Soziologie 39, 6: 493-511.

LüBbE, H. (1998): Kontingenzerfahrung und Kontingenzbewältigung. - In: Graevenitz, G. v. \& O. MaRQUARD (Hrsg.): Kontingenz. - = Poetik und Hermeneutik 17, München: Wilhelm Fink Verlag: 35-47.

Luhmann, N. (1987): Soziale Systeme. Grundriss einer allgemeinen Theorie. - Frankfurt am Main: Suhrkamp. MACY, M.W. \& J. Skvoretz (1998): The evolution of trust and cooperation between strangers: a computational model. - In: American Sociological Review 63, 5: 638-660.

Makropoulos, M. (2004): Kontingenz. Aspekte einer theoretischen Semantik der Moderne. - In: Archives Européennes de Sociologie 45, 3: 369-399.

Morgan, K. (2004): The exaggerated death of geography: learning, proximity and territorial innovation systems. - In: Journal of Economic Geography 4, 1: 3-21. MurPhy, J.T. (2006): Building trust in economic space. - In: Progress in Human Geography 30, 4: 427-450.

Parsons, T., Shils, E.A., Allport, G.W., Kluckhohn, C., Murrey, H.A., Sears, R.R., Sheldon, R.C., Stouffer, S.A. \& E.C. Tolman (2001): Some fundamental categories of the Theory of Action. A general statement. - In: PARsons, T. \& E.A. ShILs: Toward a general Theory of Action. Theoretical foundations for the social sciences. - New Brunswick, NJ: Transaction Publishers: 3-29.

Rosenberg, N. (1982): Inside the black box: technology and economics. - Cambridge: Cambridge University Press.

SHAPIRO, G.E. (1980): Is seeking help from a friend like seeking help from a stranger? - In: Social Psychology Quarterly 43, 2: 259-263.

Simmel, G. (1992): Soziologie. Untersuchungen über die Formen der Vergesellschaftung. - Frankfurt am Main: Suhrkamp. 
SombarT, W. (1987): Der moderne Kapitalismus Historisch-systematische Darstellung des gesamteuropäischen Wirtschaftslebens von seinen Anfängen bis zur Gegenwart. Erster Band. Die vorkapitalistische Wirtschaft. Zweiter Halbband. - Unveränderter Nachdruck der 2. Auflage 1916, München: Deutscher Taschenbuch-Verlag.

StichweH, R. (1992): Der Fremde - zur Evolution der Weltgesellschaft. - In: Rechtshistorisches Journal 11: 295-316.

StichweH, R. (1997): Ambivalenz, Indifferenz und die Soziologie des Fremden. - In: LuTHE, H.O. \& R.E. Wiedenmann (Hrsg.): Ambivalenz. Studien zum kulturtheoretischen und empirischen Gehalt einer Kategorie der Erschliessung des Unbestimmten. - Opladen: Leske und Budrich: 165-183.

Storper, M. \& A.J. Venables (2004): Buzz: face-toface contact and the urban economy. - In: Journal of Economic Geography 4, 4: 351-370.

Weber, M. (1999): Wirtschaft und Gesellschaft. Die Wirtschaft und die gesellschaftlichen Ordnungen und Mächte. Nachlass. Teilband 5: Die Stadt. - = Max Weber Gesamtausgabe Band 22-5, Tübingen: J.C.B. Mohr.

WeichHART, P. (2003): Gesellschaftlicher Metabolismus und Action Settings. Die Verknüpfung von Sachund Sozialstrukturen im alltagsweltlichen Handeln. - In: Meusburger, P. \& T. Schwan (Hrsg.): Humanökologie. Ansätze zur Überwindung der Natur-Kultur Dichotomie. $-=$ Erdkundliches Wissen 135, Wiesbaden: 15-44.

\section{Zusammenfassung: Der Fremde als Katalysator für Inventionen: kommunale Innovationsförderung auf Grundlage der Unbestimmbarkeit von Handlungs- folgen}

Die Wirtschaftsgeographie wendet sich in jüngerer Zeit der Interaktion zwischen Fremden zu, um Innovationsprozesse zu erklären. Der Beitrag schliesst an diese Diskussionen an und skizziert das Handeln zwischen Fremden als einen mit drei Kontingenzebenen behafteten Prozess, der für die Entstehung von Innovationen bedeutsam ist. Die erste Kontingenzebene des Handelns zwischen Fremden beruht auf der Unvoraussagbarkeit der handlungslogischen Kontinuitäten. Die zweite Kontingenzebene bildet die doppelte Kontingenz der Interaktion. Die dritte Kontingenzebene entsteht schliesslich aus der Unbestimmtheit des Fremden, die sich aus dem Sein ergibt. Der Beitrag ordnet dieses Argument in seinem zweiten Teil in den Forschungskontext ein und skizziert darauf aufbauend Möglichkeiten für die kommunale Innovationsförderung, die Fremde gezielt zusammenbringt.

Schlüsselwörter: der Fremde, Kontingenz, Handeln, Innovation, Wirtschaftsförderung

\section{Abstract: The stranger as trigger of inventions: municipal promotion of innovations on the basis of the indeterminacy of actions}

This paper is a contribution towards recent discussions in economic geography on the role of interactions between strangers in innovation processes. The essay describes interaction between strangers as a process which is affected by three different levels of contingency which affect the process of innovation. The first contingency level of foreign interaction reflects the indeterminacy of action-induced sequences. The second contingency level reflects the double contingency of interactions. Finally, the third level of contingency originates in the ontic indeterminacy of the stranger. In the second part of the paper, the interaction discussion is integrated into the current research context. Finally, possibilities for the local promotion of innovation processes are outlined with particular emphasis on bringing strangers together.

Keywords: stranger, contingency, action, innovation, economic promotion

\section{Résumé: L'étranger comme catalyseur d'inventions: promotion communale de l'innovation et imprévisibi- lité des conséquences d'action}

La géographie économique s'est récemment intéressée de manière plus intensive aux interactions entre personnes étrangères afin d'expliquer les processus d'innovation. Cet article se joint à cette discussion et décrit tout d'abord l'action entre étrangers comme un processus à trois dimensions de contingence, et significatif pour l'émergence de l'innovation. La première dimension repose sur l'imprévisibilité des continuités d'action. La deuxième dimension constitue la double contingence de l'interaction. La troisième dimension émerge de l'indétermination de l'étranger lui-même. L'article montre ensuite que cet argument peut être mobilisé à des fins de recherche pour envisager les possibilités d'une promotion de l'innovation au niveau communal qui s'appuie sur les interactions entre personnes étrangères.

Mots-clés: étranger, contingence, action, innovation, promotion économique

Dr. Peter Dirksmeier, Humboldt-Universität $\mathrm{zu}$ Berlin, Geographisches Institut, Unter den Linden 6, D-10099 Berlin, Deutschland.

e-mail:peter.dirksmeier@geo.hu-berlin.de

\section{Manuskripteingang/received/manuscrit reçu le 5.11 .2010}

Annahme zum Druck/accepted for publication/accepté pour publication: 24.6 .2011 